\title{
Éditorial
}

\section{Apport clinique de l'analyse du mouvement : évidences et perspectives}

Le mouvement est synonyme de vie. Il est primordial pour réaliser la plupart des activités de la vie quotidienne. De nombreuses pathologies affectent le mouvement. L'identification et la compréhension du mouvement déficitaire sont nécessaires pour choisir le traitement le plus adéquat. Pour ce faire, il est nécessaire de mesurer précisément le mouvement. Les méthodes utilisées aujourd'hui pour analyser le mouvement reposent sur le rapprochement des domaines de la physiologie humaine et de la mécanique qui a donné naissance à la biomécanique.

Les bases de ces connaissances ont été établies principalement entre 1500 et 1800 pour la théorie mécanique (Cardan, Euler, Newton, Descartes) et entre 1600 et 1850 pour les connaissances sur la physiologie du mouvement humain (Borelli, Van Haller, Barthez, Margendie, Poisson, Gerdy). L'application de ces connaissances pour l'analyse du mouvement a été permise par le développement de techniques de quantification du mouvement dont les deux principaux précurseurs sont J.E. Marey et E. Muydrige à qui nous devons le développement de la chronophotographie à la fin du $\mathrm{XIX}^{\mathrm{e}}$ siècle. L'intégration des techniques et méthodes d'analyse du mouvement au domaine médical commence essentiellement après la seconde guerre mondiale et concerne principalement la marche des patients amputés et atteints de poliomyélite (Perry, Elftman, Inman, Eberhardt, Ralston) et en parallèle la physiologie de la marche normale (Murray, Scherb, Taillard, Baumann). L'analyse instrumentée du mouvement se développe plus rapidement à partir des années 1980 grâce à la création de systèmes d'analyse du mouvement et à l'arrivée de l'ordinateur (plus d'informations sur l'historique du développement de l'analyse du mouvement dans les articles suivant Baker et al. (2007), Sutherland et al. $(2001,2002,2005))$. Plusieurs laboratoires d'analyse de marche s'ouvrent aux États-Unis et en Europe centrés particulièrement sur le traitement des troubles orthopédiques de la marche des enfants avec paralysie cérébrale à partir de l'analyse instrumentée de la marche prônée par le chirurgien James Gage. Cette analyse instrumentée de la marche deviendra un examen clinique à part entière appelé Analyse Quantifiée de la Marche (Clinical Gait Analysis). L'article de Carcreff et al. présente cet examen.

En France, le premier laboratoire clinique voit le jour en 1993 à Palavas les Flots (Montpellier) sous l'impulsion du chirurgien Jean-Gabriel Pous. Par la suite, plusieurs laboratoires d'analyse du mouvement se créent en France (Berck, Le Mans, Marseille, Toulouse, Bois Larris, Nancy, Lille, Lyon, Rouen, etc.). Ces laboratoires s'intéressent particulièrement à la marche pathologique. En Janvier 2001, le premier groupe français s'intéressant au mouvement humain dans un contexte clinique naît sous le nom de GAMEA (groupe d'analyse du mouvement chez l'enfant et l'adulte). La première réunion de ce groupe rassemblait à peine plus de 10 personnes à Marseille. Le groupe s'étant agrandi et le contenu étant de plus en plus scientifique, le GAMEA devient SOFAMEA en 2008 (société francophone d'analyse du mouvement chez l'enfant et l'adulte). La SOFAMEA est l'équivalent francophone de la société européenne ESMAC (European society for mouvement analysis in adults and children) et compte aujourd'hui plus de 150 membres. La SOFAMEA est ainsi un relais entre chercheurs français et européens. Elle a pour but de promouvoir la recherche dans le domaine de l'analyse du mouvement chez l'enfant et l'adulte que ce soit dans un contexte clinique, sportif ou autre. Cette société se réunit chaque année dans un congrès de trois jours autour de l'analyse du mouvement.

En 2015, le congrès de la SOFAMEA a eu lieu à Genève du 4 au 6 Février avec la thématique « Apport clinique de l'analyse du mouvement : évidences et perspectives ». Ce numéro spécial « SOFAMEA » présente 9 articles sélectionnés parmi les résumés envoyés au congrès. Ces articles reflètent l'activité de la SOFAMEA mais aussi des recherches menées dans l'analyse du mouvement dans un contexte clinique. L'enjeu final de ces recherches est de restaurer et/ou préserver le mouvement chez un patient. Pour cela, on peut identifier 3 étapes clefs (Fig. 1). 


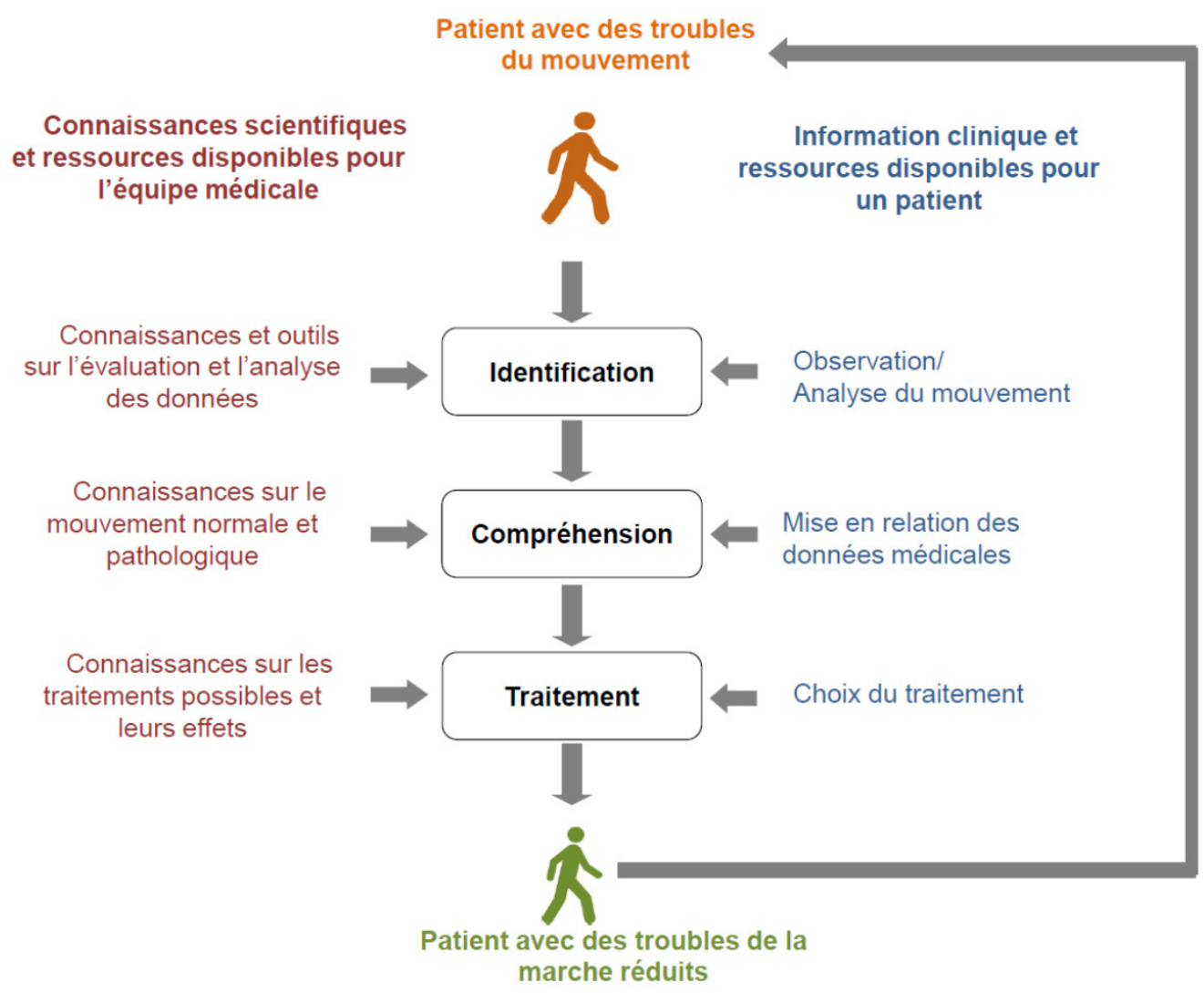

Fig. 1. Étapes clefs de prise en charge d'un patient avec des troubles du mouvement (d'après Moissenet \& Armand, 2015).

La première étape est d'identifier précisément les troubles du mouvement. Elle implique la mesure du mouvement déficitaire selon un protocole permettant de mettre en exergue ces déficits. La deuxième étape est de les comprendre. Pour cela, il est nécessaire de mettre en lien les déficits identifiés avec les causes possibles (données médicales, données issues du mouvement, imagerie). Il est aussi nécessaire de distinguer les déficits primaires et des compensations mises en place par le patient. La dernière étape est de choisir le traitement le plus approprié qui dépend de la connaissance de l'effet des traitements sur les déficits identifiés mais aussi de nombreux autres facteurs tels que l'expérience du corps médical, les attentes du patient, les outils à disposition, les risques potentiels, le délai de rééducation, le coût, etc.

Cette sélection d'articles peut ainsi être classée selon ces trois étapes clefs de la prise en charge du patient avec des troubles du mouvement. Ces articles améliorent respectivement la connaissance nécessaire aux trois étapes.

Pour améliorer l'identification des troubles du mouvement, il est possible d'améliorer les techniques et la précision des mesures, les protocoles d'évaluation ou encore les méthodes d'analyse. Trois articles illustrent ce domaine. Le $1^{\text {er }}$ article écrit par Portero et al. propose d'utiliser des capteurs inertiels (accéléromètres et gyroscopes) pour évaluer les mouvements de la tête. Cet article présente une méthode et sa reproductibilité, et évalue sa faisabi- lité en utilisation clinique chez un patient. Le $2^{\mathrm{e}}$ article écrit par Marchiori et al. s'intéresse au développement d'une méthode pour identifier les risques de développement de troubles musculo-squelettiques chez les patients en fauteuil roulant. La montée de trottoir est proposée comme tâche dans le protocole d'évaluation et le mouvement est lié à un index d'inconfort articulaire. Le $3^{\text {e }}$ article écrit par Bourrelier et al. propose d'utiliser le mouvement réalisé lors d'un lever/assis de chaise comme un biomarqueur de la fragilité chez la personne âgée.

Le deuxième domaine concerne la compréhension du mouvement non pathologique et pathologique. En effet, la compréhension du mouvement non pathologique est nécessaire à la compréhension du mouvement pathologique. Van Hamme et al. s'intéressent au développement de la marche humaine et à l'influence de la vitesse de marche. Le mouvement est très dépendant de sa vitesse de réalisation. Ainsi, comprendre comment la vitesse interagit avec le mouvement est fondamental. Schreiber et al. analysent les mouvements de la tête suivant la vitesse de marche. L'approche expérimentale est privilégiée par les chercheurs pour comprendre le mouvement humain mais d'autres approches sont également possibles telles que la simulation, la robotique, l'extraction de connaissances à partir de grandes quantités de données (data mining). L'article de Moissenet et al. montrent comment la 
simulation permet d'estimer ce qui se passe à l'intérieur du corps humain lors du mouvement et donne un nouvel éclairage pour comprendre la force axiale de compression du fémur pendant la marche. Santos et al. proposent d'utiliser les propriétés d'un robot et de programmer son déplacement avec des contraintes articulaires pour investiguer l'influence de la marche genoux fléchis et en rotation interne de hanche (situation fréquente chez les patients avec une paralysie cérébrale). Dans l'analyse des troubles du mouvement, il est toujours difficile de différencier les déviations primaires des compensations mises en jeu et de faire le lien entre les déviations et les données cliniques. Une méthode expérimentale qui permet de répondre à ces deux difficultés est d'isoler et manipuler les données cliniques. Attias et al. ont construit un exosquelette capable de répliquer des rétractions musculaires aux principaux groupes musculaires du membre inférieur chez le participant asymptomatique. Ils présentent la reproductibilité de la méthode centrée sur les muscles soléaire et triceps. L'évaluation de la marche avec des rétractions émulées à un groupe musculaire permettra de mieux comprendre les déviations primaires observées dans les rétractions musculaires et de mieux distinguer déviations et compensations.

Le troisième et dernier domaine concerne le choix du traitement et pour cela il est nécessaire d'avoir identifié et compris les troubles du mouvement mais aussi de connaître l'effet attendu du traitement sur les troubles du mouvement. De nombreuses études comparent le mouvement pré-post traitement pour évaluer le traitement. On peut aussi se servir de ces résultats pour améliorer la compréhension sur le mouvement pathologique. L'article de Galarraga et al. propose de prédire l'effet d'une chirurgie sur la marche.

Cette sélection d'articles illustre l'étendue du domaine de l'analyse clinique du mouvement qui continue de grandir rapidement. De nouvelles perspectives s'offrent aux chercheurs et cliniciens pour mieux identifier et comprendre les troubles du mouvement. Le développement de systèmes ambulatoires basés sur des capteurs inertiels permet la mesure du mouvement en dehors du laboratoire. L'utilisation des capteurs présents dans les appareils de la vie quotidienne (téléphone, montre, etc.) permet d'avoir des quantités gigantesques de données sur le mouvement des personnes. L'amélioration des modèles biomécaniques ainsi que la fusion de données entre l'imagerie et la capture 3D du mouvement permettent d'aller plus loin dans l'identification et compréhension des troubles. Des simulations basées sur des modèles neuromusculo-squelettiques permettront certainement bientôt de prédire l'effet du traitement sur les troubles du mouvement d'un patient. La mise en relation des données par des techniques avancées tels que le data mining permettront certainement d'améliorer nos capacités à comprendre les mouvements déficitaires. L'analyse clinique $\mathrm{du}$ mouvement est en plein essor et a un avenir fort prometteur.

\section{Bibliographie}

Baker, R. (2007). The history of gait analysis before the advent of modern computers. Gait \& Posture, 26 (3), 331-342.

Moissenet, F., \& Armand, S. (2015). Qualitative and quantitative methods of assessing gait disorders. In F. Canavese \& J. Deslandes (Ed.), Orthopedic management of children with cerebral palsy : A comprehensive approach. Nova Science.

Sutherland, D.H. (2001). The evolution of clinical gait analysis part 1 : kinesiological EMG. Gait 8 Posture, 14 (1), 61-70.

Sutherland, D.H. (2002). The evolution of clinical gait analysis. Part II-kinematics. Gait \&S Posture, 16 (2), 159-179.

Sutherland, D.H. (2005). The evolution of clinical gait analysis part III-kinetics and energy assessment. Gait 8 Posture, 21 (4), 447-461.

\section{Stéphane ARMAND ${ }^{1}$ et Christian BEYAERT ${ }^{2}$}

${ }^{1}$ Laboratoire de Cinésiologie, Hôpitaux Universitaires de Genève et Université de Genève, Genève, Suisse

${ }^{2}$ Président de la SOFAMEA, Laboratoire de Physiologie, Faculté de Médecine de Nancy, Nancy, France 